\title{
An Efficient Method of Image-Sound Conversion Based on IFFT for Vision Aid for the Blind
}

\author{
Xuan Zhang, Jian-Ming Wang, Xiao-Jie Duan, and Yu-Kuan Sun
}

\begin{abstract}
The vOICe technology is a kind of vision aid technology for the blind, which offers the experience of live camera views through image-sound conversion. To reduce the computational complexity of image-sound conversion, a novel image-sound conversion method is proposed in the paper. The new method takes each column of an image as the Discrete Fourier Transform (DFT) of an audio signal, and inverse Fast Fourier Transform (IFFT) is utilized to implement the inverse DFT and calculate the converted audio signal. The final result represent that this method is more effectively and has a better real time performance.
\end{abstract}

Index Terms - The vOICe system, image-sound Conversion, direct synthesis technique, inverse fast fourier transform (IFFT).

\section{INTRODUCTION}

Vision is the most important way for human to perceive the outside world, more than $80 \%$ of the information we obtain is derived from vision. The visually impaired has difficulty to gain information from eyes because of the impairment, meanwhile it also gives living or economic burden to the families and the whole society.

Broadly speaking, the tactilestick is one of the first visual aids for the blind. But with the rapid development of science and technology, people began to look for other ways to solve the problem. This paper studies the image-sound conversion method in a non-semantic way[1]: that means that image data is mapped into auditory signal without doing image semantic extraction. In this scenario, the users need special training before he or she can receive environment information from the auditory signals.

In this section, we make a brief review on the non-semantic image-sound conversion.

1) In 1992, the Dutch scholar Meijer proposed the classic method of non-semantic image [2] (also known as "Piano Transform"). Afterword, the Meijer group developed a visually impaired assistant system according to the "Piano Transform" conversion principle which is called vOICe.

2) The research group led by Dr Walker from Georgia Institute of Technology designed a device called "Listen2dRoom" [3], which use sound to help the visually impaired learn a strange room layout, and then to enhance their independent activities ability indoor [4].

3) Fang Zhi-Gang, Xu Jie etc. from Zhejiang University proposed the blind walking auxiliary system models

Manuscript received June 15, 2013; revised September 2, 2013.

The authors are with School of Electronics and Information Engineering, Tianjin Polytechnic University, Tianjin, China (e-mail: 641856373@qq.com). called "Auto Man" and "Auto Guide". Auto Man model first obtain pavement obstacles locations and path direction information by using image processing techniques, and then change parameters by the mapping such as the strength and rhythm of the sound, finally achieve the purpose of passing on information [5]. Auto Guide uses GPS and map information database technology on the basis of Auto Man for auxiliary blind walking [6].

4) Tian Ya-Nan, who is from Northeastern University, utilize edge detection and image segmentation to process visual image, and then map to voice patterns, which reduce the difficulty of the vOICe system [7], [8].

Among them, the vOICe system has the advantage of simple and convenient, thus it has a range of applications in the practical field. However, it also suffers the problem of computational complexity when real system design is concerned. To tackle the problem, a image-sound conversion method is proposed in the paper, which adopts inverse fast Fourier transform to implement the image-sound conversion more efficiently.

\section{The IMAGE-SOUnd CONVERSION METHOD AdOPTED BY THE VOICE SYSTEM}

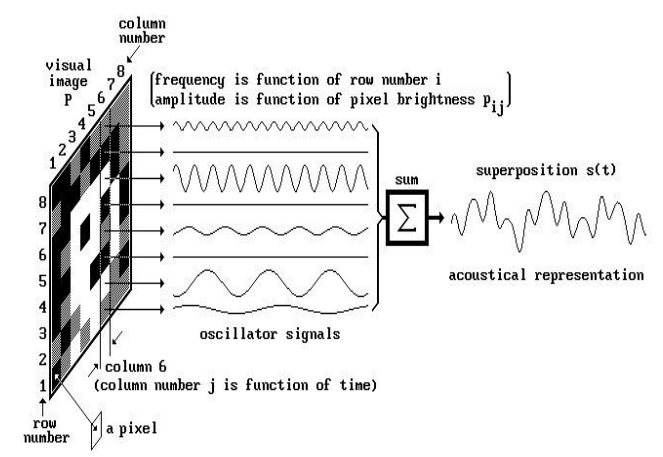

Fig. 1. The image-sound conversion principle diagram of vOICe system.

In the vOICe system, image is usually divided into a $n \times n$ pixels matrix. In the mapping of the image- sound conversion, we process one column as a unit from left to right [9]. In the transformation of each column, each pixel is to be mapped to a sine wave, which uses the mechanism of top to bottom. The frequency of the sine wave value is determined by the location of that pixel in its own column. The higher the position is, the higher the frequency. As shown in Fig. 1, the bigger the pixel number of rows (row number), the greater the frequency value. The amplitude value of the sine wave depends on the gray levels of pixels, range of $0-255$, in which white (255) corresponding to the biggest value of 1 , black (0) corresponds to the minimum value of 0 . The column number expresses the duration of the 
voice mapped from the image, the more the number of columns is, the longer the length of time. Overlying the sine waves of each pixel in a line, we will get the mapping result of one column. Then output mapping results each column arranged from left to right one by one, ultimately get the final mapping results of the whole image.

In the process of mapping, the image is divided into a $N \times$ $N$ matrix. The mapped time domain signal of the column $(i)$ is expressed with $(n)$, where $n$ indicates time $t$. Formula is as follow:

$$
\begin{aligned}
& f^{i}(n)=a_{1} \cos w_{1} n+a_{2} \cos w_{2} n+ \\
& \cdots+a_{m} \cos w_{m} n+\cdots+a_{N} \cos w_{N} n
\end{aligned}
$$

where $m$ is representative of row number of the pixel $(m=1,2, \cdots N) . a_{1} a_{2} \cdots a_{m} \cdots a_{N}, \omega_{1} \omega_{2} \cdots \omega_{m} \cdots \omega_{N}$, $n_{1} n_{2} \cdots n_{m} \cdots n_{N}$ respectively represent the amplitude value, frequency value and duration time of the sine wave mapped from each column.

According to the mapping principle of the vOICe, the greater the number of rows is, the greater the frequency. Setting

$$
w_{m}=w_{1}+\frac{w_{N}-w_{1}}{N} \times m
$$

Writing (2-1) in the summation form:

$$
f^{i}(n)=\sum_{m=1}^{N} a_{m}^{i} \cos \left(w_{1}+\frac{w_{N}-w_{1}}{N} \times m\right) n
$$

In the formula, $a_{m}^{i}$ denotes pixel values in the column $\mathrm{m}$ and row $j, w_{1}$ and $w_{N}$ separately signify the minimum and maximum frequency values of the column. Because there is not objective influence to the results, we delete $w_{1}$ in the trigonometric functions. While $w_{N}-w_{1}$ is signed with $K$ as a constant. So the formula (2-3) turns into:

$$
f^{i}(n)=\sum_{m=1}^{N} a_{m}^{i} \cos \frac{K m n}{N}
$$

\section{The PRINCIPLE OF THE NOVEL IMAGE-SOUND CONVERSION METHOD}

DFT is a kind of point to point mapping, which means sampling circularly from a continuous signal in time domain to get a set of values, and then expressing these values with frequency information in the frequency domain. In the image-sound conversion process, the sine signal mapped from each pixel of the image based on the mapping principle of the vOICe serves for the frequency domain information in the DFT calculations. Therefore, this article considers using inverse discrete Fourier transform (IDFT)to simulate the direct synthesis process in the vOICe system.

The expression of the IDFT time domain signal $x(n)$ is defined [10] as:

$$
x(n)=\frac{1}{N} \sum_{m=0}^{N-1} X(m) e^{j 2 \pi m n / N}
$$

where $X(m)$ is the sampling value of the frequency domain discrete sequence, $N$ is the total number of sampling values in the discrete sequence, " $e$ " is the base of natural logarithm, " $j$ " is the sign of imaginary number $(j=\sqrt{-1})$.

According to the Eulerformula:

$$
e^{-j \varphi}=\cos \varphi-j \sin \varphi
$$

The formula (3-1) is equal to the following:

$$
\begin{aligned}
& x(n)= \\
& \frac{1}{N} \sum_{m=0}^{N-1} X(m)[\cos (2 \pi m n / N)+j \sin (2 \pi m n / N)]
\end{aligned}
$$

where $x(n)$ is the $n t h$ IDFT output, $n$ is the index of output sequence of IDFT in the time domain $(n=0,1,2, \ldots, N-1)$, $X(m)$ is the input sample sequence, $\mathrm{m}$ is the index of input sample sequence in the frequency domain $(m=0,1,2, \ldots, N-1)$, $N$ is the number of the frequency points in the input sequence and also the sample points of IDFT output sequence.

Because the coefficient $\frac{1}{N}$ is in a linear relationship of $x(n)$, it will not affect the essence of the data in the result, only fluctuates on the amplitude, thus ignore it. Reject the imaginary part in (3-3) and the formula turns into:

$$
\begin{aligned}
& x(n)=\sum_{m=0}^{N-1} X(m) \cos (2 \pi m n / N) \\
& =X(0) \cos (2 \pi \times 0 \times n / N) \\
& +X(1) \cos (2 \pi \times 1 \times n / N) \\
& +\cdots+X(N-1) \cos [2 \pi \times(N-1) \times n / N]
\end{aligned}
$$

Compared the formula (3-4) with the direct synthesis expression of vOICe system (2-1) and (2-2), we found them with similar structure. So the process of directly superposition after point to point mapping in the vOICe system can be replaced by the inverse discrete Fourier transform (IDFT).

On the grounds of the direct synthesis expression (2-1), when transform one image, it needs $n$ times additive operations, $2 n$ times multiplications and $\mathrm{n}$ times trigonometric function calculations (Assuming that the image consists of $n \times n$ pixels). While IDFT needs $4 n$ times multiplications and $n$ times trigonometric function calculations. Seen from this, the amount of calculation of these two methods is almost consistent.

In order to reduce the amount of calculation and improve the real-time performance of the system effectively, this article uses the fast algorithm of inverse discrete Fourier transform-inverse fast Fourier transformation (IFFT) to implement the improvement on the original algorithm. 
Presume the sequence we have taken for is $N$ points of finite length, if $N$ is large enough, the ratio of the amount of calculation by usingDFT and FFT is asfollows:

$$
\frac{N^{2}+N(N-1)}{\frac{N}{2} \log _{2} N+N \log _{2} N} \approx \frac{2 N^{2}}{\frac{N}{2} \log _{2} N} \approx \frac{N^{2}}{\frac{N}{2} \log _{2} N}
$$

Suppose $N=1024$, the ratio of the calculation of DFT and FFT is 205.

Therefore we can see that utilizing the IFFT to replace direct synthesis process of the vOICe system can effectively improve the operation speed, reduce the computational complexity, and improve the real-time performance of the system.

\section{ALGORITHM IMPLEMENTATION AND EXPERIMENTAL RESULTS}

According to the principle of the vOICe system, each pixel is mapped for a sine wave with a certain frequency, that is, the frequency domain information of the pixels. To transform the frequency domain signal of one column through IFFT, point to point, we can get the time domain signal corresponds to the column. Arrange the output of each column in line with the column number from left to right, and then get the final mapping results of the whole image.

When improving the direct synthesis method of the vOICe system with IFFT, the specific process is mainly divided into the following several steps, as shown in Fig. 2.

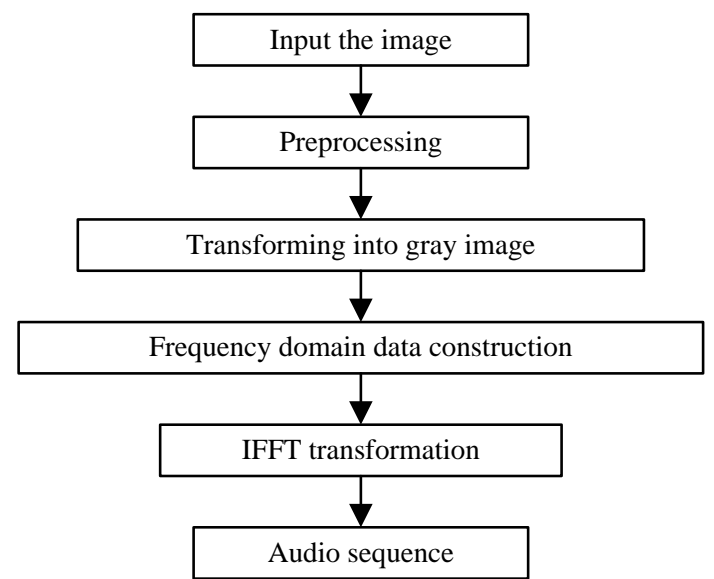

Fig. 2. Image-sound transformation algorithm improved by IFFT block diagram.

1) Input the image

2) Preprocessing:

Enhance the input image, eliminate noise etc.

3) Transforming into gray image:

Turn the image after preprocessing into grayscale image.

4) Frequency domain data construction:

Treat the gray level image as a matrix of $n \times n$. According to the mapping principle of the vOICe system, map each pixel of a unit to a sinusoidal wave of certain frequency and amplitude.

5) IFFT transformation:

Conduct he sine waves mapped from the pixels, that is, the frequency domain information, with IFFT to get the result made up of real part and imaginary part. In order to draw near to the result of the vOICe system as far as possible, according to theoretical analysis, discard the imaginary part and leave the real part to generate the final result.

6) Audio sequence:

Store the time domain data gained from the former step into wav format to form the audio sequence.

During the experiment, we convert the same image with two methods--the direct synthesis of the vOICe system and inverse fast Fourier transform (IFFT).

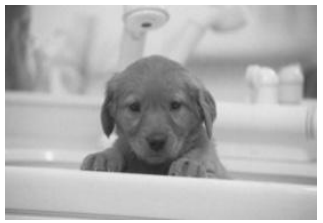

(a)

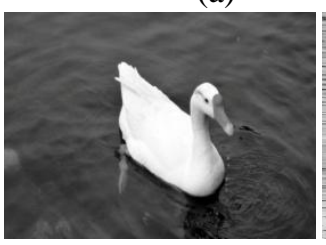

(c)

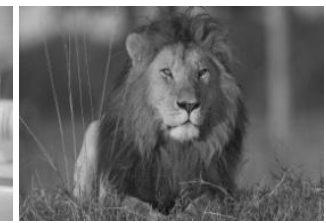

(b)

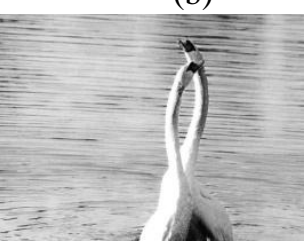

(d)
Fig. 3. Experimental images.

The pictures in Fig. 3 are all in JPG format. Because the image column numbers are too great, in this paper we choose one column every 20 ranks. The processing time of each image is shown in the following Table I.

TABLE I: THE PRocessing TIME OF THE TwO METHODS

\begin{tabular}{ccccc}
\hline \hline $\begin{array}{c}\text { File } \\
\text { name }\end{array}$ & $\begin{array}{c}\text { Image } \\
\text { size }\end{array}$ & $\begin{array}{c}\text { Direct } \\
\text { synthesis(s) }\end{array}$ & IFFT (s) & ratio \\
\hline a & $400 \times 256$ & 8.597890 & 0.129040 & 66 \\
& & & & \\
$\mathrm{~b}$ & $800 \times 512$ & 33.238021 & 0.188059 & 176 \\
$\mathrm{c}$ & $1800 \times 1024$ & 114.715937 & 0.479983 & 239 \\
$\mathrm{~d}$ & $3889 \times 2048$ & 808.205980 & 2.558285 & 315 \\
\hline \hline
\end{tabular}

Based on the formula (3-5), when the number of pixels in each column is respectively256, 512, 1024, 2048, the calculation ratio of DFT and FFT is approximately 64, 114, 205, 372 times. And the direct synthesis method and IDFT are quite proximate on amount of calculation. Therefore, the ratio of the processing time is similar to the theoretical value where we can find in Table I, which suggests that in the transformation of a image with the two methods, the IFFT transformation needs a shorter processing time than traditional vOICe conversion system.

After accumulated experiments, we find that the eventually sound generated from the same image via IFFT and the vOICe system have the same change trend, but a slightly different frequency range, while it does not affect the user to distinguish sound change trend, so it does not influence the results.

\section{CONCLUSION}

As a classical vision aid method for the visually impaired, the vOICe systemis is based on the non-semantic visual images, and it adopts the image-sound conversion method known as "Piano Transform". In this paper, the equivalence 
of IFFT and the signal synthesis process of the "Piano Transform" is proved firstly. And then we proposed a modified image-sound conversion method for improving the real-time of the vOICe system and reducing the calculated quantity. In our method, IFFT is used to realize the synthesis of mapped signals for reducing the calculation of the transformation process. At last, the method is tested on the experiment effectively, and the result has shown that the new method can decrease the conversion time exceedingly.

\section{REFERENCES}

[1] V. Pradeep, "Piecewise planar modeling for step detection using stereo vision," ECCV2008, pp. 9-15, Oct. 2008.

[2] L. Meijer, "An experimental system for auditory image representations," IEEE Transactions on Biomedical Engineering, vol. 39, no. 2, pp. 112-121, 1992.

[3] K. Davison, H. Suh, and N. Walker, "Math GNIE: Visually impaired students creating graphs and number lines on a computer," in Proc. the AER2012, Bellevue, pp. 18-22, Jul. 2012.

[4] M. Jeon, O. Nazneen, A. Ayala-Acevedo, and N. Walker, "Listen2dRoom: Helping blind individuals understand room layouts," in Proc. ACM SIGCHI Conference on Human Factors in Computing Systems, Austin, USA, May 2012, pp. 1577-1582.

[5] J. Xu, Z. G.Fang, F. L. Bao, and L. H. Zhang, "Audioman: Design and implementation of electronic travelaid,"Journal of Image and Graphics, vol. 12, no. 7, pp. 1249-1253, 2007.

[6] J. Xu, Z. G. Fang, and Z. Feng, "Sonification-Based electronic aid system for the visually impaired," Journal of Convergence Information Technology, vol. 6, no. 5, pp. 209-217, 2011

[7] Y. N. Tian, H. W. Lei, and X. Wang, "An automatic navigation system based on SVM image segmentation for the blind," Journal of Northeastern university natural science, vol. 31 , no. 12, pp. $1681-1684,2010$.

[8] Y. N. Tian, "A vision substitution method for the blind based on image edge detection and sound mapping," in Proc. Third International Conference on Computational Intelligence, Communication Systems and Networks, Shenyang, China, 2011, pp. 209-212.

[9] F. Ribeiro, D. Florêncio, P. A. Chou, and Z. Y. Zhang, "Auditory augmented reality: Object sonification for the visually impaired," in Proc. Multimedia Signal Processing (MMSP), 2012 IEEE 14th International Workshop, 2012, pp. 68-73.
[10] Lyons and G. Richard, "Understanding digital signal processing," Pearson Education, pp.167-308, 2010.

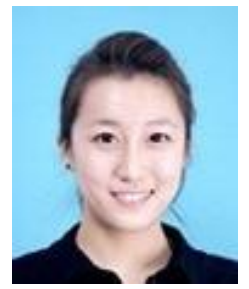

Xuan Zhang was born in 1989. She has graduated from Nankai University Binhai College, she received the College of bachelor's degree in Electronic and Information Engineering. She is now studying at Tianjin Polytechnic University of Communication and Information System for postgraduate degree, and her main research is on the intelligent image processing, machine vision and image-sound conversion.

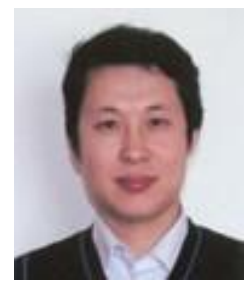

Jian-Ming Wang was born 1974. He received the Ph.D. degree in electrical engineering from Tianjin University in 2003. In 2004, he was a visiting scholar at Queens University of Belfast. He was a visiting researcher during 2007 and 2008 at Carnegie Mellon University. Since 2010, he has been an full professor in electronic information engineering at Tianjin Polytechnic University. His research interests include electronic system design, signal processing, and computer vision and pattern recognition.

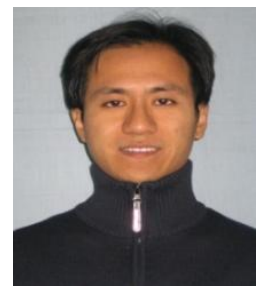

Xiao-Jie Duan was born 1980. He is a lecturer in the school of Electronics and Information Engineering in Tianjin Polytechnic University, China. He obtained his $\mathrm{PhD}$ from Tianjin University in 2013. His main research direction is photoelectronic testing technology, vision inspection.

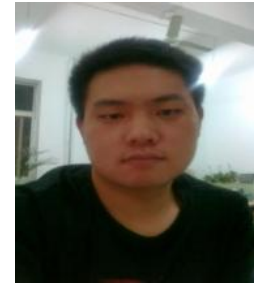

Yu-Kuan Sun was born in 1986. He has graduated from Tianjin Polytechnic University, and received the College of bachelor's degree in Electronic and Information Engineering. He is now studying at Tianjin Polytechnic University of school of Electronics and Information Engineering for postgraduate degree, and his main research is on intelligent image processing, computer vision and industrial control system. 